overall MMR status when age 50 was used as a hypothetical testing threshold. After correcting for tumour grade as a confounding variable it was shown that MLH1 and PMS2 expression were negatively correlated with increasing age while MSH6 expression was positively correlated with increasing age at diagnosis (figures 1 and 2).

Conclusion There is no statistically significant difference in overall immunohistochemical MMR status when using the age of 50 as a threshold for tumour analysis. Such a threshold would have missed $82.3 \%$ of cases with tumoral MMR deficiency and should not be included in lab protocols for EEC IHC analysis. Reflex testing of all EEC cases is highly advised as IHC testing is no longer solely about diagnosis of Lynch syndrome. Prospective evidence is required to clarify the role IHC scoring and semi-quantitative analysis should play in MMR status interpretation and patient management especially in the ever-evolving field of targeted therapeutics.

Disclosures This study was self-financed. Authors declare they have no conflict of interest, financial or otherwise.

\section{RECURRENCE AND SURVIVAL AFTER LAPAROSCOPY VERSUS LAPAROTOMY IN EARLY STAGE ENDOMETRIAL CANCER: FOLLOW-UP FIVE YEARS AFTER A RANDOMISED TRIAL}

${ }^{1}$ Bianca Reijnties, ${ }^{1}$ Geertruida H De Bock, ${ }^{1}$ Mieke Van Suijlichem, ${ }^{2}$ Arjan A Kraaijenbrink, ${ }^{3}$ Lasse Paulsen, ${ }^{4}$ Nathalie Reesink-Peters, ${ }^{5}$ Jorien Woolderink, ${ }^{6}$ Marlies Bongers, ${ }^{7}$ Mirjam Apperloo, 'Marian JE Mourits. ${ }^{1}$ University Medical Center Groningen; University of Groningen; Gynaecologic Oncology; ${ }^{2}$ Rijnstate Ziekenhuis; Gynaecology; ${ }^{3}$ Wilhelmina Hospital Assen; Gynaecology; ${ }^{4}$ Medical Spectrum Twente Enschede; Gynaecology; ${ }^{5}$ Martini Hospital Groningen; Gynaecology; ${ }^{6}$ Maxima Medical Centre Veldhoven; Gynaecology; ${ }^{7}$ Medical Centre Leeuwarden; Gynaecology

\subsection{6/ijgc-2020-ESG0.215}

Introduction/Background Laparoscopic hysterectomy has been proven to be a safe surgical procedure and is globally accepted as the standard treatment for early-stage endometrial cancer, despite insufficient data on long-term survival. Aim was to provide the five-years outcomes of a randomised Dutch trial on total laparoscopic hysterectomy (TLH) versus total abdominal hysterectomy (TAH) in early-stage low-risk endometrial cancer.

Methodology Follow-up of a multi-centre, randomised controlled trial on TLH versus TAH without routine lymphadenectomy. A total of 279 women with stage I endometrial cancer were enrolled between 2007-2009 in a 2:1 randomisation to undergo either TLH $(n=185)$ or TAH $(n=94)$. Primary outcome was disease-free survival. Secondary outcomes were primary site of recurrence, overall and disease-specific survival. The Kaplan-Meier survival curves and Cox proportional hazard ratios were applied.

Results Follow-up data of 253/279 patients are available. At a median follow-up time of 5.0 years, disease-free survival was 90.4\% after TLH and $83.3 \%$ after TAH, HR 0.68 (95\% CI 0.31-1.49). There were no port-site metastases and local recurrence rates were comparable. After adjustment for the covariates, overall survival outcomes were comparable between groups HR 0.64 (95\% CI 0.33-1.26). Disease-specific survival was comparable between both groups.
Conclusion This is the first study reporting on survival among women with early-stage endometrial cancer randomised to TLH or TAH, without routine lymphadenectomy. No significant differences were found in disease-free, overall and disease-specific survival five-years postoperatively. This supports the widespread use of laparoscopic hysterectomy as primary treatment procedure for early-stage, low-grade endometrial cancer.

Disclosures None.

\section{PREDICTING LYMPHEDEMA ASSOCIATED WITH LYMPH NODE DISSECTION IN PATIENTS WHO UNDERGO SURGERY FOR ENDOMETRIAL CANCER: ROLE OF DEMOGRAPHIC AND CLINICAL CHARACTERISTICS}

${ }^{1}$ Nazila Shokri, ${ }^{1}$ Atefeh Moridi, ${ }^{1}$ Yekta Parsa, ${ }^{2}$ Shahin Rezazadeh, ${ }^{1}$ Maryamsadat Hosseini, ${ }^{3}$ Farah Farzaneh, ${ }^{1}$ Masoumeh Raoufi. 'Shahid Beheshti University of Medical Sciences; Obstetrics And Gynecology; ${ }^{2}$ Imam Khomeini Hospital Urooncology Research Center; ${ }^{3}$ Preventative Gynecology Research Center, Obandgyn Dep; Shahid Beheshti University of Medical Sciences

\subsection{6/ijgc-2020-ESG0.216}

Introduction/Background Multimodal treatments have significantly improved oncological outcomes in patients with endometrial cancer. Therefore most of the patients are longterm survivors and may experience adverse effects related to treatment. Lymph node dissection in patients who undergo total abdominal hysterectomy with bilateral salpingo-oophorectomy (TAH+BSO) may be associated with adverse effects such as lymphedema. Several factors including number of removed lymph nodes and extent of lymph node dissection have been postulated to be associated with adverse events. However, in most studies the definition of the complications is poorly described and contradicting findings exist. In this prospective study we aim to assess the complications related to lymph node dissection in patients who undergo $\mathrm{TAH}+\mathrm{BSO}$ and potential factors that predict adverse events.

Methodology We conducted this prospective study to assess the complications related to lymph node dissection in patients who underwent $\mathrm{TAH}+\mathrm{BSO}$ for endometrial cancer. Patients with prior history of lower limb surgery, pelvic radiation and prior history of cancer treatment were excluded from study. Additional exclusion criteria were heart failure, uncontrolled thyroid abnormalities and other disorders associated with impaired lymphatic drainage and/or lower limb edema.Demographic and clinical characteristics including age, body mass index, pathologic stage, and extent of lymph node dissection were recorded. We also assessed complications related to lymph node dissection i.e. lymphedema, lymphatic leakage and lymphocele. Lymphedema was defined based on American Physical Therapy Association criteria. We applied Gynecologic Cancer Lymphedema Questionnaire to evaluate severity of symptoms related to lymphedema. Written informed consent was obtained from all patients and institutional review board approved the study.

Results A total of 135 patients with a mean age of 57.6 \pm 11.5 underwent $\mathrm{TAH}+\mathrm{BSO}$ during the study period. Lymph node dissection was performed in $83(61.4 \%)$ patients. The extent of lymph node dissection was limited to pelvis in 18 (33.3\%) 
patients, whereas, $3(2.2 \%)$ patients underwent pelvic and para-aortic lymph node dissection. Median number of dissected lymph nodes was 11 (interquartile range: 5.7-21.2). Among patients who underwent lymph node dissection, 14 (16.3\%) patients showed lymph node involvement. Stage I, II and III were recorded in 97(71.9\%), 13 (9.6\%), and 25 (18.5\%) patients respectively. We did not observe lymphedema in our study participants. Other complications related to lymph node dissection were low grade and were not associated with age, BMI, extent of lymph node dissection, total number of dissected lymph nodes, lymph node involvement and disease stage.

Conclusion Complications related to lymph node dissection including lymphedema are rare after $\mathrm{TAH}+\mathrm{BSO}$ for endometrial cancer and the extent of lymph node dissection or disease stage is not associated with higher risk of such complications.

\section{CLINIC-PATHOLOGICAL FEATURES OF MIXED ENDOMETRIAL CARCINOMA; EVALUATION OF 29 PATIENTS IN A SINGLE TERTIARY CENTER}

Mete Sucu, Omer Faruk Geckil, Umran Kucukgoz Gulec, Ahmetbaris Guzel, Ghanim Khatib, Mehmet Ali Vardar. Cukurova University Faculty of Medicine; Obstetrics and Gynecology

\subsection{6/ijgc-2020-ESG0.217}

Introduction/Background Mixed endometrial carcinoma (MEC) refers to a tumor that is comprised of two or more distinct histotypes. Each component histotype by definition has to represent more than $5 \%$ of the tumor. Although it is relatively rare, both diagnosis and management can be troublesome. Molecular and histopathologic features have become important in the identifcation and more importantly the precise management of the MEC's.

In our study, we aimed to evaluate the clinical and pathological characteristics of the MEC

Methodology The clinical and pathological records of the 29 MEC patients who were operated on and regularly followed up in the clinic between January 2000-December 2019 were reviewed. Clinical features, operation characteristics, pathological findings, myometrial invasion degree (MI), lymph node involvement (LNI), lymphovascular space invasion (LVSI), adjuvant therapies, and follow-up data of the patients and their effects on survival were investigated.

Results During the study period, 29 out of 1110 patients with endometrial cancer had MEC (2.6\%). Eighteen of the cases had endometrioid + serous, 7 had endometrioid + clear, 3 had endometrioid + serous, and 1 had clear + serous histopathology. The mean age of the patients was $63.2 \pm 12.1$. Laparoscopic surgery was performed in 8 of the cases $(27.6 \%)$. Sixteen of the cases were in stage $1(55.1 \%), 4$ were in stage $2(13.8 \%)$, and 9 were in stage $3(31 \%)$. LVSI was positive in 17 cases (58.6\%). LNI was detected in 7 cases (24.1\%). Approximately 80 percent of cases received adjuvant therapy. While $80 \%$ of the cases received chemotherapy, this rate was $55 \%$ for radiotherapy.

Conclusion MECs are tumors that can be difficult to diagnose and manage. In addition to histopathological features, revealing and evaluating their molecular properties will help us to better understand this group of tumors.

Disclosures None

\section{Miscellaneous}

\section{IMPACT OF THE COVID PANDEMIC ON GYNAECOLOGICAL CANCER SURGERY - RESULTS FROM THE COVIDSURG GYNAECOLOGICAL CANCER INTERNATIONAL STUDY}

${ }^{1}$ Sudha S Sundar, ${ }^{1}$ Elaine Leung, ${ }^{2}$ Tabassum Khan, ${ }^{1}$ James Glasbey, ${ }^{3}$ Nadeem Abu-Rustum, ${ }^{4}$ Luis M Chiva, ${ }^{5}$ Anna Fagotti, ${ }^{6}$ Keiichi Fujiwara, ${ }^{7}$ Rahel Ghebre, ${ }^{8}$ Murat Gultekin, ${ }^{9}$ Thomas Konney, ${ }^{10}$ Joseph Ng, ${ }^{11}$ Rene Pareja, ${ }^{12}$ Rajkumar Kottayasamy Seenivasagam, ${ }^{13}$ Jalid Sehouli, ${ }^{14}$ Ts Shylasree, ${ }^{1}$ Aneel Bhangu, ${ }^{15}$ Christiana Fotopoulou, ${ }^{16}$ Covidsurg Cancer Collaborators. 'University of Birmingham; Institute of Cancer and Genomic Sciences; University of Birmingham; ' University of Birmingham; Nihr Global Health Research Unit on Global Surgery; ${ }^{3}$ Memorial Sloan Kettering Cancer Center; ${ }^{4}$ Clínica Universidad de Navarra; ${ }^{5}$ Catholic University of the Sacred Heart; Fondazione Policlinico A. Gemelli; ${ }^{6}$ Saitama Medical University; International Medical Center; ' University of Minnesota; Department of Obstetrics, Gynaecology and Women's Health; ${ }^{8}$ Hacettepe University Faculty of Medicine; Department of Obstetrics and Gynaecology; ${ }^{9}$ Kwame Nkrumah University Of Science and Technology; Komfo Anokye Teaching Hospital; ${ }^{10}$ National University Cancer Institute; Division of Gynecologic Oncology; ${ }^{11}$ Clinica de Oncología Astorga; Gynaecologic Oncology; ${ }^{12}$ All India Institute of Medical Sciences; Obstetrics and Gynaecology; ${ }^{13}$ Charité Universitätsmedizin Berlin; Klinik für Gynäkologie; Charité Universitätsmedizin Berlin; ${ }^{14}$ Tata Memorial Hospital; Department of Gynecologic Oncology and Mdt; ${ }^{15} /$ mperial College London; ${ }^{16}$ Nihr Global Health Research Unit

\subsection{6/ijgc-2020-ESG0.218}

Introduction/Background Covid-19 has resulted in significant number of elective surgeries being delayed or cancelled worldwide with an estimated 28 million patients being affected. ${ }^{1}$

Studies show that perioperative Covid-19 infection has a high perioperative mortality of $23 \cdot 8 \%$. (2) Complications increase with any additional treatment burden such as cytotoxic chemotherapy, radiotherapy or immunotherapy. ${ }^{3}$ In an effort to reduce treatment related morbidity and mortality during the Covid-19 pandemic, many elective anticancer treatments have been postponed or modified. ${ }^{4}$

Methodology We investigated the impact of the Covid-19 pandemic on gynaecological cancer surgery in an international prospective multi-centre study. Participating centres entered consecutive patient's data into a customized electronic database for 12 weeks from the first COVID positive patient managed in their hospital between March and June 2020. Patients were eligible for enrollment if they were planned to undergo surgery during the study duration, regardless of COVID-19 status and whether they underwent surgery as recommended or not. Patients who did not undergo their planned surgery were followed up for 12 weeks to observe outcomes. Descriptive analysis of outcomes is presented.

$4490 / 4472(95 \%)$ patients received surgery; of these $17 \%$ $(n=758)$ experienced change or adaptation of surgery. The main impact was on surgical timing; $11 \%(n=483)$ experienced delay in surgery, 3\% $(n=119)$ a change in choice of operation, $10 \% \quad(\mathrm{n}=452)$ received surgery in alternative hospital.

Patients in this study had confirmed resolved COVID-19 prior to surgery in $0.95 \%(n=45)$ patients with an additional $0.34 \%(n=16)$ with probable resolved COVID-19 infection. A post-operative COVID-19 rate of $2.27 \%(n=25)$ and pulmonary complication rate of $1.8 \%(n=20)$ was found in the initial analysis of the Covidsurg cancer data, analysing outcomes for 1102 gynaecological cancer patients. The overall 30-day mortality rate in this cohort was $1.18 \%(n=13) .^{5}$

Conclusion The largest multi-centre analysis of gynaecological cancer surgery during the Covid-19 pandemic has 